\title{
VASCULAR PATHOLOGY OF AN EYE AND THE THREAT OF DEVELOPING AGE-RELATED MACULAR DEGENERATION AFTER RADIATION EXPOSURE
}

\author{
Pavlo Fedirko \\ Laboratory of MIT \\ State Institution "National Research Center for Radiation Medicine of \\ the National Academy Medical Sciences of Ukraine" \\ 53 Melnykova str., Kyiv, Ukraine, 04050 \\ Vasiliy Sakovich \\ Department of Neurology and Ophthalmology \\ State Institution "Dnipropetrovsk Medical Academy of the Ministry of health of Ukraine" \\ 9 Volodymyra Vernadskogo str., Dnipro, Ukraine, 49044 \\ Natalia Garkava \\ Department of Neurology and Ophthalmology \\ State Institution "Dnipropetrovsk Medical Academy of the Ministry of health of Ukraine" \\ 9 Volodymyra Vernadskogo str., Dnipro, Ukraine, 49044
}

\begin{abstract}
On the contrary to the previous expectations, the most common Chornobyl catastrophe victim's pathology was the vascular pathology of an eye. The work is devoted to the assessment of changes in capillary tubes, arterioles and venules, which were irradiated as a result of Chornobyl catastrophe and those who have been exposed to radiation while working in the Chornobyl exclusion zone.

The results of eye vascular system examination is presented by 615 persons: 358 Chornobyl clean-up workers, inspected in 1993-1994, 2007-2008 and 257 participants of works at the «Shelter» object, that were inspected in 2007-2008 and in 2017.

Microcirculatory damages in conjunctiva are the most early ionizing radiation induced eye damage. Dose dependence from microcirculatory damages in conjunctiva has been proved. The conjunctiva index, which characterizes the evaluation degree of microcirculatory abnormalities value for individuals irradiated at a dose of up to $10 \mathrm{mSv}$ was $5.33 \pm 0.34$, at a dose of 10 to $30 \mathrm{mSv}-9.71 \pm 0.93$. Thus, a significant $(\mathrm{t}=4.42, \mathrm{p}<0.05)$ increase in the display of microcirculatory abnormalities in conjunctiva was revealed with an increase in the dose load. In the correlation analysis, there was found a direct connection, $\mathrm{r}=0.48$, between the conjunctiva index and the dose of irradiation.

The expressive tendency to correlate the negative dynamics of the macular zone with the degree of deterioration of microcirculation shows the effect of microcirculatory disorders on the development of age-related macular degeneration. At the correlation analysis, there was found a direct connection of significant decreased number of functioning capillaries with the deterioration of the macular zone of the retina $(\mathrm{r}=0.37)$. It is also advisable to appoint prophylactic complexes of lutein and zeoxanthine to patients in the case of microcirculatory disorders.
\end{abstract}

Keywords: ionizing radiation, eye, dose, microcirculatory damages, conjunctiva, age-related macular degeneration.

\section{Introduction}

Traditionally, the main effect of ionizing radiation pathology of an eye was considered the radiation cataract [1]. Studies devoted to radiation cataract research still urgent [2]. However, earlier there was not much attention given to other irradiated eye pathological changes, such as changes in accommodation [3], violation of refractogenesis in children [4], change the skin of an eye [5] ets, due to a small amount of health data in period to the Chornobyl catastrophe. As a result of the Chornobyl disaster, the observations were held of large irradiated groups, the most common pathology was the vascular pathology of an eye [6]. Changes in retinal vessels were discovered by us and other authors in the first years after the Chernobyl catastrophe [7]. They develop over time after radiation exposure [8]. The great interest is to examine microcirculation in the bulbar conjunctiva, because the abnormalities in microcirculation could be one of the first signs of radiation exposure [9]. It 
was also shown that the microcirculation in the conjunctiva of participants of emergency works in Chornobyl clean-up in 1986 in the distant period was significantly violated [9].

In this paper, we classified the dose-effect correlation for capillaries, arterioles, and venules of the bulbar conjunctiva, that were irradiated as a result of the Chornobyl disaster and those who have been exposed to radiation while working in the Chornobyl exclusion zone. Accordingly, there is a possibility to estimate occurrence time of changes in microcirculation after radiation exposure.

In the rest of the rock, the boule is brought, but macular degeneration is one of the most potent risk pathology of radiological attack [10].

The pathogenesis of age-related macular degeneration is small known, same hypothesis development in last decades [11]. The observation that age-related macular degeneration and atherosclerosis share risk factors and pathogenetic mechanisms has led to the development of a hypothesis that is identified as a haemodynamic or vascular model of the pathogenesis of AMD [12]. It holds that AMD is a vascular disorder, characterized by impairment of choroidal perfusion of the retinal pigment epithelium. This model, suggested by Friedman [12], was updated to incorporate reported evidence that the changes affecting Bruch's membrane in age and AMD involve lipoproteins processed by the retinal pigment epithelium. The Friedman model proposes that [12] these lipoproteins accumulate in drusen and in Bruch's membrane because the choriocapillaries does not clear them. This model asserts that scleral rigidity and choroidal vascular resistance is increased by decreased compliance of ocular tissue as a result of progressive infiltration with lipid. The systemic circulation is the source of the lipid in the sclera and choroidal vasculature, there is evidence that the retinal pigment epithelium is the source of lipids in drusen and in Bruch's membrane. The vascular model contends that impairment of choroidal perfusion, consisting of decreased blood flow and elevated hydrostatic pressure, compromises the processing of outer segment lipid by the retinal pigment epithelium and the clearance of the lipoproteins secreted by the retinal pigment epithelium. This results in drusen, pigment changes, and geographic atrophy, as well as calcification and fracture of Bruch's membrane. Progressive accumulation of hydrophobic lipids in Bruch's membrane may compromise retinal pigment epithelium function further by decreasing its hydraulic conductivity. The model [12] proposes that the combination of elevated choriocapillary pressure breaks in Bruch's membrane, and vascular endothelial growth factor causes choroidal neovascularization.

Our work evidences that the pathology of the retina vessels is the most common pathology of an eye in individuals after radiation exposure, its development can be expected in 4-5 years after exposure. The main risk factors for its development in Chornobyl clean-up workers' cohort are age and dose of external irradiation. Primary morbidity for angiopathy reached its first maximum in 9 years after radiation exposure. The second lift of angiopathy is in 13 years [13].

Therefore, the study of the association of microcirculatory disturbances in the bulbar conjunctiva and changes in the macular zone in subjects exposed to ionizing radiation is of great practical importance.

\section{Aim}

The aim of the study is investigate microcirculatory disturbances in the bulbar conjunctiva in subjects exposed to ionizing radiation and association microcirculatory disturbances in the bulbar conjunctiva and of changes in the macular zone of the retina in subjects exposed to ionizing radiation.

\section{Methods}

We used the examination results of the 1993-1994 and 2007-2008 people, who had any contact with ionizing radiation.

Survey results of 338 clean-up workers at the Chornobyl NPP in 1986 (1993-1994 - the period of the survey), aged from 27.7 to 73.37 years old, the average age was $46.91 \pm 0.53$ years. The dose of irradiation is known for 210 of them, it ranged from 0.0003 Gy to $2.20 \mathrm{~Gy}$, the average dose was $0.253 \pm 0.023 \mathrm{~Gy}$. The control group consisted of 110 industrial workers who did not have any contact with the IR (ionizing radiation) aged from 26.7 to 62.1 years old during the examination. 
In 2007-2008, 201 persons were examined; they were the ones, who worked at the Shelter facility (2006-2008), and 20 participants, Kyiv citizens of different age, who were clean-up workers at the Chornobyl NPP in 1986. The age of the surveyed participants at the "Shelter" ranged from 62.04 to 19.57 years old; the average age was $38.9 \pm 0.9$ years old. Dose loads for the work period are currently estimated for 20 people, the doses ranged from 0.3 to $29 \mathrm{mSv}$, the average dose was $12.87 \pm 0.52 \mathrm{mSv}$. The average age of people, who were clean-up workers in Chornobyl for the survey realized in $2007-2008$ is $53.85 \pm 0.68$ years old. The average absorbed dose of ionizing radiation (for the whole body) was $0.395 \pm 0.032 \mathrm{~Gy}$. Thus, the dose loads of the one liquidation team member were incomparably large (the equivalent of the average absorbed dose of a participant at the "Shelter" facility is less than $0.013 \mathrm{~Gy}$ ).

In 2017, 56 people, who worked at the "Shelter" facility, were inspected, their age ranged from 23 to 48 years.

The studies of microcirculation in the conjunctiva were carried out by vessels biomicroscopy of the bulbar conjunctiva using a slit lamp SL115 Zeiss, Germany, zooming the image up to 20 times. The image was recorded using a web camera and a software package from Zeiss, Germany, further increased by $200 \%$, after that it was evaluated and processed. To improve the intensity of the vascular pattern, the color scale of the image was applied, as well as, identification of the vessels of the bulbar conjunctiva. Also they allocated a zone of radially located, parallel and not anastomosing channels, the middle zone, consisting of several anastomoses, and the area of terminal capillaries towards sulcus sclera.

An assessment of the microcirculatory canal was carried out according to the classical qualitative and quantitative scheme for the evaluation of microcirculation indices in the conjunctiva of L. Malloy, etc. They studied the caliber of microvessels, their course, tortuosity, arterial-venous ratio, the number of functioning capillaries, the size of the avascular regions, the background transparency degree, the presence of venous sacculations, microaneurism, perivascular edema, hemorrhage, and sludge phenomenon. Each characteristic was evaluated in points, the conjunctiva index (CI) was calculated, which is normally 0 points and the maximum possible number of points in pathology is 33 [9].

In 2017 a comparative study of the dynamics of microcirculation (caliber of microvessels, arterial-venous ratio, the number of functioning capillaries, the size of the avascular regions, the background transparency degree, the presence of venous sacculations, microaneurism, sludge phenomenon, perivascular edema, hemorrhage) and the dynamics of the degree of manifestation of initial dystrophic changes in the retina macular zone (characteristic for AMD) according to the analysis of images received on the fundus camera was realized.

The results were based on the statistical evaluation of the average amount of quantitative indicators, dispersion or root-mean-square deviation, asymmetry coefficients. Criteria statistics (t) was used in the comparative analysis using the Student method. They studied the connection between these factors using the correlation analysis with the evaluation of the correlation coefficient and its margin of error.

\section{Results}

In the study of bulbar conjunctiva with participants, who weren't irradiated, a parallel arrangement of arterioles and venules was found, the ratio of their diameters ranged from $1 / 2$ to $2 / 5$, the boundaries of the vessels were equal and the blood flow was continuous, fine-grained, the conjunctiva was transparent.

In the examined group (1993-1994) of clean-up workers there was found a significant prevalence of microcirculation abnormalities. The pathology of the microcirculatory bed most often occurred in the form of vascular pathology: vascular tortuosity, uneven venule caliber and sometimes arterioles, spastic arterioles, dilated venules until venous suctions appeared in many of these cases with decreased arteriovenous ratio, decreased number of functioning capillaries, and appearance of microaneurysms. Intravascular abnormalities were represented by a sludge phenomenon: blood flow was hampering and in some cases to the extent of stasis. 
There were also perivascular changes - opacification of the background, edema, microhemorrhagia, pigment deposition. The conjunctiva index (CI), which characterizes the evaluation degree of microcirculatory abnormalities in the group of liquidation team members was $12.05 \pm 0.27$, in the control group - 3.60 \pm 0.91 . Compared with the control group, conjunctiva index of clean-up workers' group was significantly higher $(\mathrm{t}=8.9, \mathrm{p}<0.001)$. At the correlation analysis, there was found a direct probable $(\mathrm{p}<0.01)$ ratio connecting with age $(\mathrm{r}=0.22 \pm 0.05)$ and a documented dose of external irradiation $(\mathrm{r}=0.16 \pm 0.06)$

The average $\mathrm{CI}$ in the group of clean-up workers with radiation dose up to $10 \mathrm{cGy}$ was $11.58 \pm 0.47$, for persons with a radiation dose of $10-25 \mathrm{cGy}-12.43 \pm 0.86$, with a dose rate of $25-50 \mathrm{cGy}-13.13 \pm 0.73$, with a dose load of 50 or more $\mathrm{cGy}-14.53 \pm 1.09$. It was established that for individuals with a dose load of $25 \mathrm{cGy}$ and more, the CI was significantly higher than for those irradiated at a dose of up to $10 \mathrm{cGy}(\mathrm{t}=2.00, \mathrm{p}<0.05)$.

The conjunctiva index varied from $10.5 \pm 2.88$ for participants under the age of 30 to $12.71 \pm 0.74$ for $50-59$ year olds and $13.92 \pm 1.65$ for those whose age is 60 years and older. It is known that a number of common diseases, in particular, diabetes mellitus, hypertensive disease, ischemic heart disease are accompanied by a significant abnormality of microcirculation. Taking into account the presence of a connection between the value of CI and the level of blood pressure, on the basis of the results it is impossible to conclude whether the microcirculation abnormality is the direct result of the influence of IR, or it is caused by the influence of other pathological changes and related to the dose load indirectly. To clarify this issue, a comparative study has been carried out concerning microcirculation in the group of liquidation team members with different dose loads without hypertension, diabetes, ischemic heart disease, retinal dystrophy and glaucoma at the age of 30-39 and 40-49 years old. The data of the quantitative assessment of the state of microcirculation showed that the cause of changes in microcirculation was largely the irradiative impact.

Hemocirculation studies (2007-2008) of the people, who took part in the transformation of the "Shelter" facility into an environmentally safe system, showed that the average CI in the examined group was $7.53 \pm 0.07$. Dependence analysis of changes in microcirculation in the conjunctiva upon effect from radiation doses received during the period of stabilization works, showed a tendency for an increase in the coefficient characterizing the degree of microcirculation abnormality with an increase in the dose load. The average coefficient value for individuals irradiated at a dose of up to $10 \mathrm{mSv}$ was $5.33 \pm 0.34$, at a dose of 10 to $30 \mathrm{mSv}-9.71 \pm 0.93$. Thus, a significant $(\mathrm{t}=4.42$, $\mathrm{p}<0.05$ ) increase in the display of microcirculatory abnormalities in conjunctiva was revealed with an increase in the dose load. At the correlation analysis, there was found a direct connection between the $\mathrm{CI}$ and the dose of irradiation $(\mathrm{r}=0.48)$ (Fig. 1).

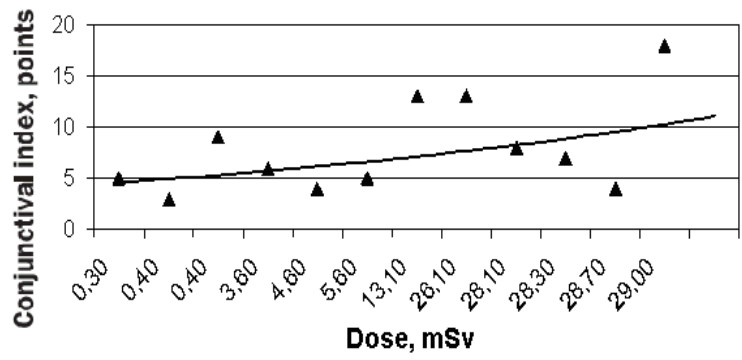

Fig. 1. Dependence of changes in the coefficient, which characterizes the degree of violation of microcirculation in the conjunctiva, points, from the radiation dose, $\mathrm{mSv}$

As expected, the coefficient characterizing the degree of microcirculation abnormalities in the conjunctiva tended to increase with the age of the examinees. Its average value (in the group of participants with known dose load) was $6.33 \pm 0.59$ for participants under the age of $40,9.14 \pm 0.89$ for participants aged 40 and over $(\mathrm{t}=4.42, \mathrm{p}<0.05)$. At the correlation analysis, there was found a direct, but weak CI connection with the age of the examinees $(r=0.29)$.

The revealed tendencies have been preserved in the examination group of 2017. At the same time, it was found that deterioration of the macular zone of the retina, an increase in the volume 
of lesions of dyspigmentation, druzes, according to the analysis of images obtained on the fundus camera, has a clear tendency to correlate with the degree of deterioration of microcirculation. At the correlation analysis, there was found a direct connection of significant decreased number of functioning capillaries with the deterioration of the macular zone of the retina $(r=0.37)$.

The expressive tendency to the correlation between the negative dynamics of the macular region of the retina and the degree of deterioration of microcirculation shows the effect of microcirculatory disorders on the development of AMD.

In the survey of 2007-2008 in the group of clean-up workers a significant prevalence of microcirculation abnormalities was found. The pathology of the microcirculatory bed occurred not only in the form of vascular pathology: vascular tortuosity, uneven caliber of venules and arterioles, spastic condition of arterioles, dilatation of veins, venous suctions, lowering ratio of arteriovenous, decrease in the number of functioning capillaries, appearance of microaneurysms: intravascular abnormalities were represented by a sludge phenomenon: blood flow was hampering and in some cases to the extent of stasis. There were also perivascular changes - background opacification, edema, microhemorrhagia, pigment deposition. Clinically, there was an increased frequency of organic changes in microvessels. In 1993-1994 years the contribution of functional changes was even larger. The conjunctiva index, which characterizes the degree of microcirculatory abnormalities, was $11.94 \pm 0.39$ in the liquidation team group members with known dose loads examined 22 years after the radiation exposure. Thus, the average CI for clean-up workers from 1994-1995 has not been increased. With the correlation analysis in 2007-2008 there was discovered a direct connection of the CI with the age of the examined participants $(\mathrm{r}=0.19)$ and a documented dose of external irradiation $(\mathrm{r}=0.42)$. The conjunctiva index varied from $11.13 \pm 0.67$ for group of former clean-up workers up to 50 years old to $12.0 \pm 0.91$ for those whose age was 50 years or more. But the difference between the age groups was not statistically significant $(\mathrm{t}=0.76)$.

It was established that in the groups of clean-up workers at the Chornobyl NPP with a dose load from 10 to $200 \mathrm{cGy}$, in 2007-2008 conjunctiva index was $15.10 \pm 0.82$, which is significantly higher $(\mathrm{t}=8.9, \mathrm{p}<0.001)$ in comparison with former clean-up workers at the Chornobyl NPP with a dose load from 2 to 10 cGy (conjunctiva index during this period $-6.57 \pm 0.38$ ).

The risk of macular degeneration development increases in groups of radiation-infected persons [14], and changes in the frequency of vascular pathology occurred earlier in these groups [15]. It is known that the use of antioxidants inhibits the development of age-related macular degeneration [16]. Food disturbances contribute to the development of age-related macular degeneration [17], and the presence of sufficient amounts of lutein and zeaxanthin in the diet reduces the risk of age-related macular degeneration [18]. Systematic review and meta-analysis of several longitudinal studies have concluded that lutein and zeaxanthin affect positively in the case of late AMD but not early AMD [19]. Our data clearly showed that additional use of lutein and zeoxanthin in the form of vitamin complexes helps to stop the development of age-related macular degeneration in radiation exposed [20].

It is also advisable to appoint prophylactic complexes of lutein and zeoxanthine (for example, Superoptic [21] ets.) to patients in the event of microcirculatory disorders.

\section{Discussion}

The data obtained indicate a significant damage to the microvasculature of the vascular system during radiation exposure. At the same time, microcirculation abnormalities occurred early - practically in the first months after the radiation exposure.

Changes in microcirculation in the groups of clean-up workers at the Chornobyl NPP after the radiation exposure in 8 to 9 years were more essential, but the dose loads were significantly higher $(0.253 \pm 0.023 \mathrm{~Gy}$, while the equivalent of the average absorbed dose of the participant at the "Shelter" facility was less than 0.013 Gy). Therefore, it is possible that the degree of microcirculation abnormalities after the first year of radiation exposure is practically not increasing. This assumption is confirmed by the fact that the conjunctiva index in clean-up workers was not higher 22 years after the radiation exposure than in $8-9$ years after irradiation. There was only an increase in the weight of organic changes. 
The expressive tendency to the correlation of the negative dynamics of the macular region of the retina with the degree of deterioration of microcirculation shows the effect of microcirculatory disorders on the development of AMD in radiation exposed persons. The vascular model, by emphasizing the pathogenetic importance of atherosclerotic processes and increased intravascular pressure, essentially predicts that statins and antihypertensive agents etc. should have a protective effect on AMD [12]. Therefore, further research in this direction is relevant. It is known that the use of antioxidants [16], lutein and zeoxanthin [18] inhibits the development of age-related macular degeneration.

It is also advisable to appoint prophylactic complexes of lutein and zeoxanthine (for example, Superoptic [21] ets.) to radiation exposed patients in the case of microcirculatory disorders.

\section{Conclusions}

1. The data obtained indicate a significant damage to the microvasculature of the vascular system during radiation exposure.

2. Microcirculation abnormalities occurred early - practically in the first months after the radiation exposure.

3. Dose dependence from microcirculatory damages in conjunctiva has been proved.

4. The expressive tendency to the correlation between the negative dynamics of the macular zone and the degree of deterioration of microcirculation shows the effect of microcirculatory disorders on the development of age-related macular degeneration.

5. It is also advisable to appoint prophylactic radiation exposed patients in the case of microcirculatory disorders.

\section{References}

[1] Merriam, G. R., Focht, E. F. (1957). A clinical study of radiation cataract and the relationship to dose. The American Journal of Roentgenology Radium Therapy and Nuclear Medicine, 77 (5), 759-785.

[2] Worgul, B. V., Kundiyev, Y. I., Sergiyenko, N. M., Chumak, V. V., Vitte, P. M., Medvedovsky, C. et. al. (2007). Cataracts among Chernobyl Clean-up Workers: Implications Regarding Permissible Eye Exposures. Radiation Research, 167 (2), 233-243. doi: 10.1667/rr0298.1

[3] Sergienko, N. M., Fedirko, P. (2002). Accommodative Function of Eyes in Persons Exposed to Ionizing Radiation. Ophthalmic Research, 34 (4), 192-194. doi: 10.1159/000063879

[4] Serdyuchenko, V. I., Nostopireva, E. I. (2015). Refractogenesis among schoolchildren living on a radically contaminated territory. Odessa: Astroprint.

[5] Fedirko, P. (2011). Oxygen exchange of cornea and lens of an eye: perspective of radiopatronage in the conditions of radiation influence. Problems of ecological and medical genetics and clinical immunology, 103 (1), 227-230.

[6] Buzunov, V., Fedirko, P. (1999). Ophthalmo-Pathology in Victims of the Chernobyl Catastrophe - Results of a Clinical Epidemiological Study. Ocular Radiation Risk Assessment in Populations Exposed to Environmental Radiation Contamination. Dordrecht-Boston-London: Kluwer Ac. Publish., 57-67. doi: 10.1007/978-94-011-5278-5_8

[7] Fedirko, P., Kadoshnikova, I.; Blokov, I., Sadownichik, T., Labunska, I., Volkov, I. (Eds.) (2007). Risks of eye pathology in victims of the Chernobyl catastrophe. The health effects of the human victims of the Chernobyl catastrophe. Amsterdam: Greenpeace, 16-24.

[8] Fedirko, P. A., Garkava, N. A., Krynychna, I. P. (2009). Primary retinal angiopathy incidence in liquidators of the Chornobyl disaster. Problems of ecological and medical genetics and clinical immunology, 96 (9), 490-494

[9] Fedirko, P. A., Garkava, N. A. (2016). Microcirculation violations of the conjunctiva in clean-up workers of the Chornobyl NPP accident. Problems of radiation medicine and radiobiology, 21, 345-351.

[10] Fedirko, P.; Serdiuk, A., Bebeshko, V., Bazyka, D., Yamashita, S. (Eds.) (2011). 18.1 Eye: clinic, diagnostics, regularities and risks for development of eye pathology in Chornobyl catastrophe sufferers. Health effects of the Chornobyl accident. A Quarter of century aftermath. Kyiv: DIA, 492-510. 
[11] Anderson, D. H., Mullins, R. F., Hageman, G. S., Johnson, L. V. (2002). A role for local inflammation in the formation of drusen in the aging eye. American Journal of Ophthalmology, 134 (3), 411-431. doi: 10.1016/s0002-9394(02)01624-0

[12] Friedman, E. (1997). A Hemodynamic Model of the Pathogenesis of Age-related Macular Degeneration. American Journal of Ophthalmology, 124 (5), 677-682. doi: 10.1016/s0002-9394(14)70906-7

[13] Fedirko, P. A., Garkava, N. A. (2016). Common factors of development of retinal vascular pathology in the remote period after radiation exposure. Journal of Ophthalmology, 6, 24-28.

[14] Babenko, T. F., Fedirko, P. A., Dorichevska, R. Y., Denysenko, N. V., Samoteikina, L. A., Tyshchenko, O. P. (2016). The risk of macular degeneration development in persons antenatally irradiated as a result of Chornobyl NPP accident. Problems of radiation medicine and radiobiology, 21, 172-177.

[15] Fedirko, P., Babenko, T., Dorichevska, R.; Brindza, J., Klymenko, S. (Eds.) (2016). Patterns of development and clinical features of eye diseases in radiation exposed - thirty years after Chernobyl. Biodiversity after the Chernobyl accident. Part I: The scientific proceedings of the international network AgroBioNet. Nitra: Slovak University of Agriculture in Nitra, 74-76.

[16] The Relationship of Dietary Lipid Intake and Age-Related Macular Degeneration in a Case-Control Study: AREDS Report No. 20 (2007). Archives of Ophthalmology, 125 (5), 671-679. doi: 10.1001/archopht.125.5.671

[17] Wierzbowska, J., Figurska, M., Stankiewicz, A., Sierdzioski, J. (2008). Risk factors in age-related macular degeneration and glaucoma - own observations. Klinika Oczna, 110 (10-12), 370-374.

[18] Tan, J. S. L., Wang, J. J., Flood, V., Rochtchina, E., Smith, W., Mitchell, P. (2008). Dietary Antioxidants and the Long-term Incidence of Age-Related Macular Degeneration. Ophthalmology, 115 (2), 334-341. doi: 10.1016/j.ophtha.2007.03.083

[19] Ma, L., Dou, H.-L., Wu, Y.-Q., Huang, Y.-M., Huang, Y.-B., Xu, X.-R. et. al. (2011). Lutein and zeaxanthin intake and the risk of age-related macular degeneration: a systematic review and meta-analysis. British Journal of Nutrition, 107 (03), 350-359. doi: 10.1017/s0007114511004260

[20] Fedirko, P., Babenko, T., Dorichevska, R.; Hanych, T. M. (Ed.) (2016). The effectiveness of the macular pigment and resveratrol combination in the treatment and prevention of age-related macular degeneration. Modern aspects of the preservation of human health. Uzhhorod, 287-289.

[21] Mirzabekova, K. A. (2014). Novel therapeutic approaches to correct retinal metabolic abnormalities in primary open-angle glaucoma and age-related macular degeneration. Ophthalmologia, 11 (4), 41-46. 